\title{
Advancing Materials and Methods for Photoelectrochemical Energy Conversion
}

\author{
Kevin Sivula* \\ Werner Prize 2017
}

\begin{abstract}
Strategies to convert energy from the sun into chemical fuels and feedstocks for a carbon-neutral economy are under rapid development. A photoelectrochemical route uses a direct semiconductor-liquid junction that offers simplicity but places challenging constraints on the materials used. The LIMNO lab has made significant progress in demonstrating new viable classes of materials, defining new methods to control the nanostructure of photoelectrodes to enhance charge extraction, and to engineer the electrode interfaces to reduce losses in photoelectrodes. These techniques contribute to a toolset which will enable inexpensively processed, robust semiconductor materials to obtain high performance solar to fuel conversion. Herein the major advances and achievements of LIMNO toward this goal are highlighted within the context of the current state of the art and the future prospects for the field.
\end{abstract}

Keywords: Hydrogen $\cdot$ Interface engineering $\cdot$ Nanostructure $\cdot$ Semiconductor $\cdot$ Solar fuel

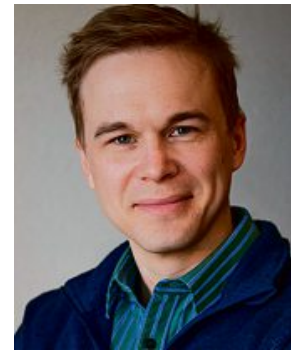

Kevin Sivula studied Chemical Engineering at the University of Minnesota and the University of California (Berkeley), obtaining his PhD in 2007 with research performed in the group of Prof. Jean Fréchet. He then joined the Laboratory of Photonics and Interfaces at the EPFL, led by Prof. Michael Grätzel, and in 2011 he accepted an appointment as tenure-track assistant professor at EPFL in the Institute of Chemical Science and Engineering. There he heads the Laboratory for

${ }^{*}$ Correspondence: Prof. Dr. K. Sivula

Ecole Polytechnique Fédérale de Lausanne (EPFL) Institut des sciences et ingénierie chimiques

Laboratory for Molecular Engineering of Optoelectronic Nanomaterials (LIMNO)

EPFL SB ISIC LIMNO, Station 6

$\mathrm{CH}-1015$ Lausanne

E-mail: kevin.sivula@epfl.ch
Molecular Engineering of Optoelectronic Nanomaterials (LIMNO), which focuses on the development of solution processed semiconductor thin films for the next generation of economical and robust optoelectronic devices and solar energy conversion.

\section{Introduction}

In the post-fossil fuel era, our society will require routes to efficiently convert renewable energy into chemical feedstocks and fuels using abundant starting materials like $\mathrm{CO}_{2}, \mathrm{~N}_{2}$, and water. The efficient electrochemical reduction of these starting materials into $\mathrm{CO}, \mathrm{CH}_{3} \mathrm{OH}, \mathrm{NH}_{3}$, and $\mathrm{H}_{2}$ can potentially form the basis for a sustainable chemical network supplying the manufacturing, agriculture, and transportation industries. ${ }^{[1]}$ Given the abundance of solar energy, it is of particular interest as a primary renewable source to power these transformations, ${ }^{[2]}$ and while traditional photovoltaic devices can be, in principle, used to drive standard electrolyzer devices, ${ }^{[3]}$ the direct integration of light harvesting and catalysts in a photocatalytic or a photoelectrochemical device that can convert sunlight directly into fuel via artificial photosynthesis is a promising approach. The Laboratory for Molecular Engineering of Optoelectronic Nanomaterials (LIMNO) at EPFL has been dedicated to the pursuit of robust light-harvesting semiconductor photoelectrodes to drive the efficient and economical artificial photosynthesis of solar fuels. In this article the major advances and achievements of LIMNO toward this goal are highlighted within the context of the current state of the art and the future prospects for the field.

\section{The Photoelectrochemical Tandem Cell}

The direct conversion of solar energy into chemical fuels can be achieved by a photoelectrochemical device, which consists of a semiconductor electrode in direct contact with a liquid electrolyte. A Schottky-type junction, which can form at the semiconductor-liquid interface generates an electric field in the semiconductor that drives the separation of photogenerated carriers (electrons and holes) produced by the absorption of photons with energy greater than the semiconductor band gap energy. ${ }^{[4]}$ The separation of carriers produces a photopotential that can drive a reduction reaction (in the case of a p-type photocathode) or an oxidation reaction (for a n-type photoanode). In the simple case of photoelectrochemical water splitting to form molecular hydrogen at the cathode and oxygen at the anode a (thermodynamic) minimum of $1.23 \mathrm{~V}$ photopotential is required (more with kinetic overpotentials). Since no one semiconductor has been identified to provide sufficient photopotential while also harvesting a sufficient portion of the solar spectrum to afford efficient solar-to-fuel conversion, and since two electrodes are required for any complete electrochemical operation, a tandem cell employing a photoanode and a photocathode that harvest complimentary portions of the solar spectrum is envisioned as a more 
viable device design. ${ }^{[5]} \mathrm{A}$ schematic of a photoelectrochemical (PEC) tandem cell for water splitting is shown in Fig. 1 and the details of the operation are summarized in the figure caption.

Identifying ideal semiconductors for the economically-viable PEC tandem cell is an ongoing challenge in the field, ${ }^{[6]}$ as materials need to satisfy band gap requirements for high conversion efficiency, ${ }^{[5 a]}$ must consist of widely available and lowcost component atoms, be inexpensive to manufacture, and be sufficiently robust to withstand continuous and long-term photoelectrochemical operation in relatively harsh conditions (in the presence of water and oxygen). Oxide semiconductors have been particularly attractive for use as photoanodes as - being already oxidized - they can potentially be stable under oxidative conditions. Our group has demonstrated tandem cells for solar hydrogen production using $\mathrm{Fe}_{2} \mathrm{O}_{3}, \mathrm{WO}_{3}$ or $\mathrm{BiVO}_{4}$ based semiconductors oxide photoanodes with solarto-hydrogen efficiencies of up to $3 \% .^{[7]}$ However, in these reports a photocathode with a direct semiconductor-liquid junction was not used given the challenge to identify stable p-type semiconductors for the water reduction reaction. Indeed, the development of photocathodes is currently a major goal in the field. Moreover the current state-of-the art performance of the commonly-employed oxide photoanodes is still not sufficient to drive solar-to-hydrogen conversion at efficiency over $10 \%$ - a benchmark taken for economical competiveness with traditional photovoltaic + electrolyzer technology. ${ }_{[}^{8]}$ Thus the development of new material systems is needed to advance the field of PEC solar fuel production. A primary focus of our research group is towards the development of semiconductor photoelectrodes that, while they are composed of robust and inexpensive semiconductors materials, can suffer from limitations in photogenerated charge harvesting. Thus our overall goal is to advance generalizable methods to enhance the performance of promising materials for PEC application.

\section{New Materials Development}

There are many strategies to advance the development of new semiconductor materials for photoelectrochemical cells. An effective strategy is to identify promising candidates from computational or combinatorial studies and investigate indepth their optoelectronic properties to determine if future work is warranted, or if the intrinsic material properties will ultimately prevent high performance. With respect to p-type photocathodes, ternary

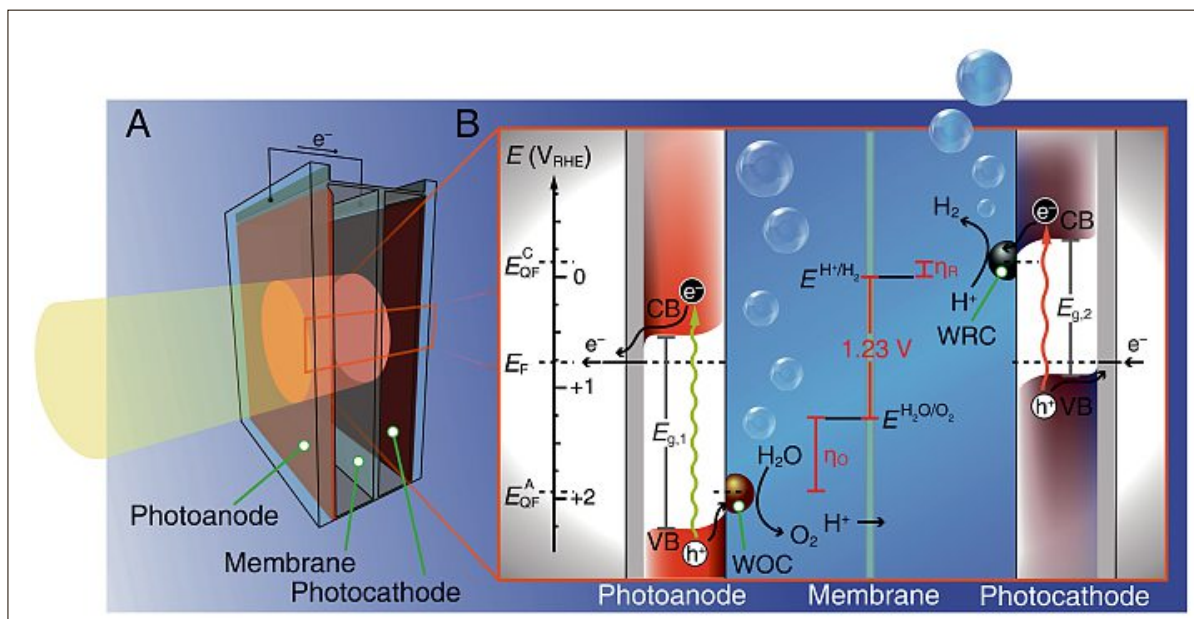

Fig. 1. PEC tandem cell schematic. (A) A 'wired-type' tandem cell for water splitting with solar illumination first incident on the photoanode and transmitting to the photocathode. Panel (B) shows the working principle of the tandem cell for water splitting using a photoanode with band gap energy $E_{\mathrm{q}, 1}$, and a photocathode with $E_{\mathrm{q}, 2}\left(E_{\mathrm{q}, 1}>E_{\mathrm{g}, 2}\right)$. Briefly, upon absorption of a solar photon, an electron (e-) from the valence band (VB) is promoted to the conduction band (CB) leaving the corresponding electron hole $\left(h^{+}\right)$. The electric field in the depletion layer physically separates these charges and, in the photocathode, the electrons in the CB drift to the SCJL, increasing the quasi-Fermi energy of the cathode, $\left(E_{Q F}^{C}\right)$, to drive the reduction of $\mathrm{H}^{+}$to $\mathrm{H}_{2}$ at a water reduction catalysis (WRC) site. Analogously in the photoanode, electron holes in the VB drift to the semiconductor-liquid interface, increasing the photoanode's quasi-Fermi energy, $\left(E_{Q F}^{A}\right)$, enough to surmount the overpotential for the oxidation $\left(\eta_{0}\right)$ and oxidise water to $\mathrm{O}_{2}$ at a water oxidation catalysis (WOC) site. Photogenerated electrons in the CB of the photoanode travel through the external circuit to recombine with the holes in VB of the photocathode. Figure adapted from ref. [6].

oxides containing copper(I) cations have emerged as a promising class of stable materials for water or $\mathrm{CO}_{2}$ reduction, through a collection of initial reports. ${ }^{[9]}$ Our group has performed extensive research and development on one of the most promising: $\mathrm{CuFeO}_{2}$ delafossite (see crystal structure in Fig. 2A). ${ }^{[10]}$ While this copper(I) iron(III) oxide material had been suggested as a promising photocathode given its suitable band edge positions and band gap energy of $1.5 \mathrm{eV},{ }^{[11]}$ difficulty with its preparation had previously limited study in PEC systems. However, our development of a simple sol-gel route ${ }^{[10 a]}$ to prepare thin film electrodes of $\mathrm{CuFeO}$, opened a path for the understanding of the optoelectronic material properties and the assessment of its viability for further development. Importantly, we found a relatively long photogenerated carrier lifetime and good carrier mobility, which together suggest that this material is intrinsically capable of high performance solar water reduction. ${ }^{[10 \mathrm{~d}]}$ Moreover, photoelectrodes were found to be exceptionally stable under operation for days. However, while we have managed to extract up to $2.6 \mathrm{~mA} \mathrm{~cm}^{-2}$ of photocurrent from $\mathrm{CuFeO}_{2}$ photocathodes under standard illumination conditions in sacrificial electrolyte, ${ }^{[10 \mathrm{~b}]}$ our work has identified a critical challenge of (likely extrinsic) surface trapping states that currently limit the performance. ${ }^{[10 \mathrm{~d}]}$

Inspiration for new materials development can come from other fields as well. The field of organic semiconductors is rich with material design given the ability to control molecular structure via organic chemistry. Our group's experience in this field ${ }^{[12]}$ aided in the recent identification of a stable organic photoanode material.[13] We used a conjugated polymer known for high stability (poly[benzimida zobenzophenanthroline], coded as BBL, see structure Fig. 2A) for the direct solar water oxidation and found that in aqueous electrolyte with a sacrificial hole acceptor $\left(\mathrm{SO}_{3}{ }^{2-}\right)$, photoelectrodes gave modest but stable photocurrents up to $0.23 \mathrm{~mA} \mathrm{~cm}^{-2}$ at $1.23 \mathrm{~V}$ vs. the reversible hydrogen electrode (RHE) under standard simulated solar illumination. ${ }^{[13]}$ While molecular $\mathrm{O}_{2}$ evolution was measured upon functionalization with a nickel-cobalt co-catalyst, smaller photocurrents were observed for water oxidation compared to the sacrificial case. This suggested a limitation in charge separation, which we found to be related to the driving force for charge injection into the electrolyte. Our work reveals that further efforts with organic semiconductors should address the free charge generation/ separation at the semiconductor-liquid interface. Importantly, this demonstration was the first to indicate that robust n-type conjugated organic semiconductors are 

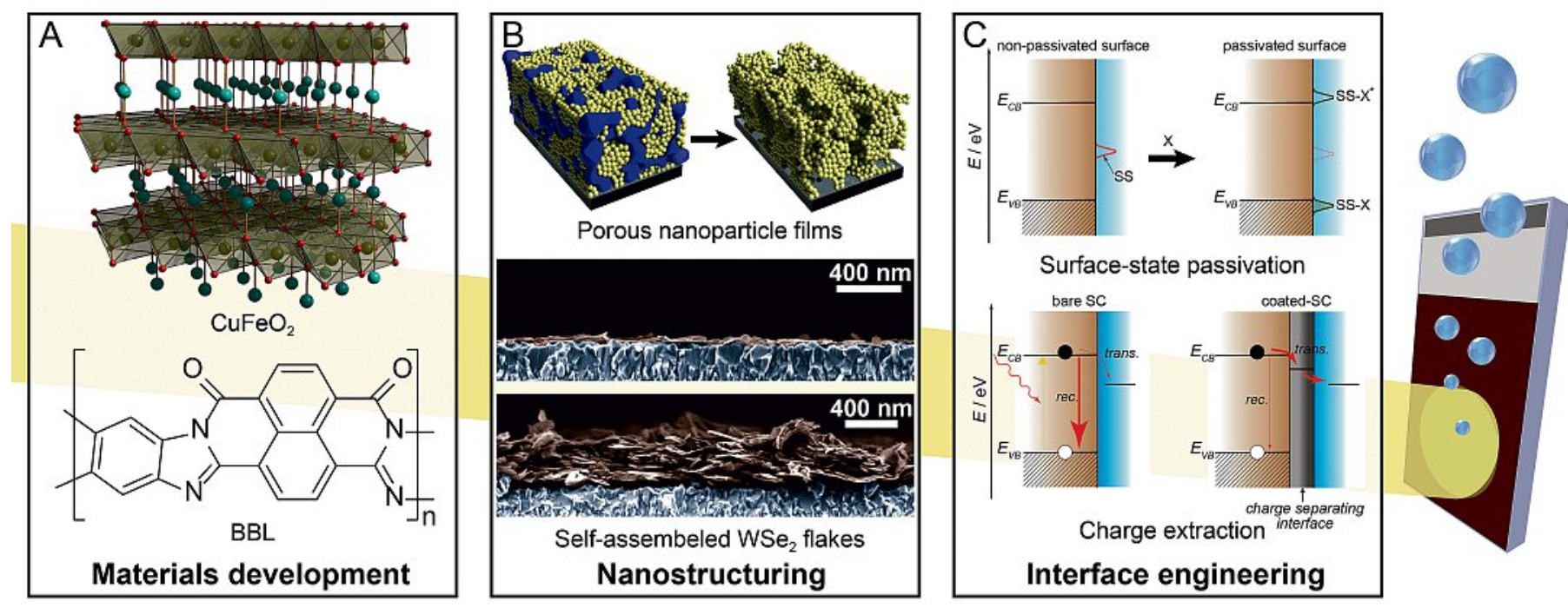

Fig. 2. Strategies to advance photoelectrochemical solar fuel production are shown. (A) The crystal structure of $\mathrm{CuFeO}_{2}$ is shown with red oxygen atoms and blue copper atoms (top) and the structure of the BBL polymer (bottom) is also shown. (B) schematic of the porogen approach for mesostructured photoelectrodes (top) and the cross-sectional SEM morphology (bottom) of $\mathrm{WSe}_{2}$ films (colored brown) on $\mathrm{F}: \mathrm{SnO}_{2}$ substrates (colored blue) prepared by the liquid/liquid (upper) and air/liquid (lower) approaches. (C) Reduction of the density surface states (SS) or passivation by reaction with acid or Lewis base reagents $(X)$ which creates new bonding (SS-X) and antibonding (SS- $\left.X^{\star}\right)$ states out of the band gap (between the conduction band energy $E_{C B}$ and valence band energy $E_{v B}$ ), (top). Enhancement of the charge separation by depositing an additional semiconducting layer that selectively accepts one of the carriers (trans. and rec. represent the electron transfer and recombination processes, respectively, and the thickness of arrow qualitatively depicts the probability of each process).

suitable for direct PEC water oxidation. It will likely open a new path for the rational design and optimization of photoanodes for solar water splitting.

\section{Nanostructuring Photoelectrodes}

One important aspect of photoelectrode development is the morphology of the semiconductor thin film. Depending on the material properties, better performance may be realized with a single crystalline thin film, or a film with a very high surface area. For example, for a material like $\mathrm{Fe}_{2} \mathrm{O}_{3}$ with a very small photogenerated carrier diffusion length $(\mathrm{ca} .10 \mathrm{~nm})$ compared to the light absorption depth (ca. $400 \mathrm{~nm}),{ }^{[14]}$ highly nanostructured films with a large interfacial area between the semiconductor and electrolyte will benefit the performance. In contrast, for materials with surface or grain boundary trapping defects, high surface areas can reduce performance.[15] While obtaining high surface area oxide photoelectrodes has been well known for many years using a nanoparticle or sol-gel plus porogen approach, ${ }^{[16]}$ the procedure requires high temperatures $\left(>400{ }^{\circ} \mathrm{C}\right)$ to oxidize the organic porogen (leaving the oxide nanoparticle scaffold behind). These conditions are not suitable for many nonoxide semiconductors like chalcogenides or pnictogens. To enable the use of these types of semiconductors for high-surface area PEC application, we developed a low-temperature nanostructuring technique using nitrocellulose as a porogen. Our method is shown schematically in Fig. 2B (top) where semiconductor nanocrystals (yellow) together with the nitrocellulose (blue) are cast into a thin film after which a mild thermal treatment of $250{ }^{\circ} \mathrm{C}$ is sufficient to decompose the porogen to afford a mesoporous film. We demonstrated this technique with $\mathrm{CdS}$ and copper zinc tin sulfide (CZTS) nanocrystal thin film electrodes with remarkable control over the surface area and the average pore size (20-100 $\mathrm{nm}$ ) by varying the ratio of nanocrystals and nitrocellulose. We found that the photoelectrochemical performance is enhanced due to the increased active surface area, showing an 8-fold photocurrent increase over compact nanocrystal films and an a quantum efficiency over $70 \%$.[17]

An emerging type of nanostructured semiconductor is based on the layered transition metal dichalcogenides, which can be exfoliated into 2D nanoflakes with promising optoelectronic properties for solar energy conversion including hydrogen production. ${ }^{[18]}$ However, the application of this class of materials is limited by the processability into large-area thin films. ${ }^{[19]}$ Recently, we developed a novel self-assembly approach for the thin film formation of solvent-exfoliated $2 \mathrm{D} \mathrm{WSe}_{2}$ nanoflakes using a liquid/liquid interface formed from two non-solvents. ${ }^{[20]}$ The superior self-assembly of the $2 \mathrm{D}$ flakes at the liquid/liquid interface compared to a traditional (liquid/air) interfacial self-as- sembly technique led to films with fewer aggregates and good contact to the substrate (see cross-sectional scanning electron microscope images in Fig. 2B, bottom). The functionalization of these films with platinum as a co-catalyst gave solar fuel production (hydrogen evolution) photocurrents over $1.0 \mathrm{~mA} \mathrm{~cm}{ }^{-2}$ under standard illumination conditions (compared to only $50 \mu \mathrm{A} \mathrm{cm}{ }^{-2}$ with an aggregated film) with device active area in the $\mathrm{cm}^{2}$ range. In contrast, the photocurrent density reported with single crystal photoelectrodes under similar conditions ${ }^{[21]}$ was about $20 \mathrm{~mA} \mathrm{~cm}^{-2}$ suggesting that more advances with charge carrier harvesting and extraction $^{[22]}$ are needed to realize high-performance solution-processed TMD-based photoelectrodes.

\section{Surface Engineering Methods}

In photoelectrochemical applications, semiconductor photoelectrodes must drive photogenerated carrier separation, catalyze the desired electrochemical reaction, and avoid recombination via charge trapping sites. Since the interface of the semiconductor and liquid electrolyte is the most critical point for these processes, engineering the interface to reduce charge carrier trapping or aid in charge extraction is often necessary to improve performance compared to only applying a co-catalyst overlayer. ${ }^{23]}$ These two processes are shown schematically in Fig. 2C. 
Our group has shown the usefulness of these approaches in a number of systems. [10a,13,15,22,24] Specifically, charge extraction overlayers are useful when the semiconductor liquid junction does not develop a sufficient electric field to drive charge separation. We have demonstrated enhanced charge separation with $\operatorname{CZTS}^{[24 a, b]}$ and related CIGS ${ }^{[15]}$ photocathodes, and interestingly, we showed that charge transfer through mid-gap states can occur with a $\mathrm{ZnSe}$ overlayer suggesting more flexibility in the energetic positions of the overlayer bands. An organic semiconductor was also used to extract charges from $\mathrm{MoS}_{2}$ photoanodes. ${ }^{[22]}$ Alternatively with respect to surface state passivation, altering the halide used for nanocrystal coalescence from $\mathrm{Cl}^{-}$to $\mathrm{I}^{-}$was found to enhance the performance even without a charge extracting overlayer due to, in part, the passivation of surface states. ${ }^{[24 c]}$ Despite the demonstrated ability to passivate surface states in a number of other photoelectrode systems, ${ }^{[23 b]}$ including $\mathrm{Fe}_{2} \mathrm{O}_{3}$ via the atomic layer deposition of $\mathrm{Al}_{2} \mathrm{O}_{3},{ }^{[25]}$ they remain a challenge in the aforementioned $\mathrm{CuFeO}_{2}$ photocathode system.

\section{Conclusions and Outlook}

The realization of economically competitive direct solar to fuel production with a photoelectrochemical device remains under development and the identification of ideal materials is still needed to enable the commercial viability of this technology. The research performed by our labs and summarized above give promise to the possibility of PEC solar fuels by demonstrating new viable classes of materials, defining new methods to control the nano- structure of photoelectrodes to enhance performance and to engineer the electrode interfaces to enhance performance. These contributions to the field help to build a set of tools with which to accelerate the development of devices toward more practical applications, giving a bright future for converting sunlight into fuel with artificial photosynthesis.

Received: June 5, 2017

[1] a) E. V. Kondratenko, G. Mul, J. Baltrusaitis, G. O. Larrazabal, J. Perez-Ramirez, Energy Environ. Sci. 2013, 6, 3112; b) A. Harriman, Phil. Trans. R. Soc. A 2013, 371, 20110415; c) F. L. Formal, W. S. Bourée, M. S. Prévot, K. Sivula, CHIMIA 2015, 69, 789.

[2] N. S. Lewis, Science 2016, 351, aad1920.

[3] A. C. Nielander, M. R. Shaner, K. M. Papadantonakis, S. A. Francis, N. S. Lewis, Energy Environ. Sci. 2015, 8, 16.

[4] N. Guijarro, F. L. Formal, K. Sivula, CHIMIA 2015, 69, 30 .

[5] a) M. S. Prévot, K. Sivula, J. Phys. Chem. C 2013, 117, 17879; b) K. Sivula, CHIMIA 2013, $67,155$.

[6] K. Sivula, R. van de Krol, Nat. Rev. Mater. 2016, 16010.

[7] a) J. Brillet, J. H. Yum, M. Cornuz, T. Hisatomi, R. Solarska, J. Augustynski, M. Graetzel, K. Sivula, Nat. Photonics 2012, 6, 824; b) P. Bornoz, F. F. Abdi, S. D. Tilley, B. Dam, R. van de Krol, M. Graetzel, K. Sivula, J. Phys. Chem. C 2014, 118, 16959.

[8] B. A. Pinaud, J. D. Benck, L. C. Seitz, A. J. Forman, Z. Chen, T. G. Deutsch, B. D. James, K. N. Baum, G. N. Baum, S. Ardo, H. Wang, E. Miller, T. F. Jaramillo, Energy Environ. Sci. 2013, 6, 1983.

[9] I. Sullivan, B. Zoellner, P. A. Maggard, Chem. Mater. 2016, 28, 5999.

[10] a) M. S. Prévot, N. Guijarro, K. Sivula, ChemSusChem 2015, 8, 1359; b) M. S. Prevot, Y. Li, N. Guijarro, K. Sivula, J. Mater. Chem. A 2016, 4, 3018; c) W. S. Bourée, M. S. Prévot, X. A. Jeanbourquin, N. Guijarro, M. Johnson, F. L. Formal, K. Sivula, Adv. Mater. 2016, 28,
9308; d) M. S. Prévot, X. A. Jeanbourquin, W. S. Bourée, F. Abdi, D. Friedrich, R. van de Krol, N. Guijarro, F. Le Formal, K. Sivula, Chem. Mater. 2017, 11, 4952.

[11] J. Gu, A. Wuttig, J. W. Krizan, Y. Hu, Z. M. Detweiler, R. J. Cava, A. B. Bocarsly, J. Phys. Chem. C 2013, 117, 12415.

[12] a) A. Gasperini, S. Bivaud, K. Sivula, Chem. Sci. 2014, 5, 4922; b) A. Gasperini, X. A Jeanbourquin, A. Rahmanudin, X. Yu, K. Sivula, Adv. Mater. 2015, 27, 5541; c) X. A. Jeanbourquin, A. Rahmanudin, A. Gasperini, E. Ripaud, X. Yu, M. Johnson, N. Guijarro, K. Sivula, J. Mater. Chem. A 2017, 5, 10526.

[13] P. Bornoz, M. S. Prévot, X. Yu, N. Guijarro, K. Sivula, J. Am. Chem. Soc. 2015, 137, 15338.

[14] K. Sivula, F. Le Formal, M. Grätzel, ChemSusChem 2011, 4, 432.

[15] N. Guijarro, M. S. Prévot, X. Yu, X. A. Jeanbourquin, P. Bornoz, W. Bourée, M Johnson, F. Le Formal, K. Sivula, Adv. Energy Mater. 2016, 6, 1501949.

[16] K. Sivula, R. Zboril, F. Le Formal, R. Robert, A. Weidenkaff, J. Tucek, J. Frydrych, M. Grätzel, J. Am. Chem. Soc. 2010, 132, 7436.

[17] N. Guijarro, M. S. Prévot, X. A. Jeanbourquin, X. Yu, K. Sivula, Chem. Mater. 2015, 27, 6337.

[18] X. Yu, K. Sivula, ACS Energy Lett. 2016, 1, 315.

[19] X. Yu, M. S. Prévot, K. Sivula, Chem. Mater. 2014, 26, 5892.

[20] X. Yu, M. S. Prevot, N. Guijarro, K. Sivula, Nat. Commun. 2015, 6, 7596.

[21] J. R. McKone, A. P. Pieterick, H. B. Gray, N. S Lewis, J. Am. Chem. Soc. 2012, 135, 223.

[22] X. Yu, A. Rahmanudin, X. A. Jeanbourquin, D. Tsokkou, N. Guijarro, N. Banerji, K. Sivula, ACS Energy Lett. 2017, 2, 524.

[23] a) K. Sivula, J. Phys. Chem. Lett. 2013, 4, 1624 ; b) N. Guijarro, M. S. Prevot, K. Sivula, Phys. Chem. Chem. Phys. 2015, 17, 15655.

[24] a) L. Rovelli, S. D. Tilley, K. Sivula, ACS Appl Mater Interfaces 2013, 5, 8018; b) N. Guijarro, M. S. Prévot, K. Sivula, J. Phys. Chem. Lett. 2014, 5, 3902; c) N. Guijarro, M. S. Prévot, M. Johnson, X. Yu, W. S. Bourée, X. A. Jeanbourquin, P. Bornoz, F. L. Formal, K. Sivula, J. Phys. D: Appl. Phys. 2017, 50, 044003.

[25] F. Le Formal, N. Tetreault, M. Cornuz, T. Moehl, M. Gratzel, K. Sivula, Chem. Sci. 2011, 2, 737. 